\title{
False detection of dangerous and neutral substances in commonly used materials by means of the standard THz Time Domain Spectroscopy
}

\author{
V. A. Trofimov \\ vatro@cs.msu.ru
}

\section{S. A. Varentsova}

Lomonosov Moscow State University, Leninskiye Gory, Moscow, Russia, 119992

Lomonosov Moscow State University, Leninskiye Gory, Moscow, Russia, 119992

Essential limitations of the standard THz Time Domain Spectroscopy (TDS), which lead to false detection of dangerous and neutral substances in commonly used materials, are demonstrated using the physical experiment with chocolate under real conditions as well as with semiconductors under laboratory conditions. To overcome this disadvantage, we propose using the time-dependent spectrum of the THz pulse, transmitted through or reflected from a substance. For quality assessment of the standard substance absorption frequency presence in the signal under analysis, we use time-dependent integral correlation criteria. The influence of aperture placed in front of the sample on spectral properties of silicon wafers with different resistivity is demonstrated as well.

[DOI: http://dx.doi.org/10.2971/jeos.2016.16016]

Keywords: Transmitted and reflected few-cycle THz pulse, Time-Domain Spectroscopy, method of spectral dynamics analysis (SDA-method), detection and identification of substances, integral correlation criteria

\section{INTRODUCTION}

As it is well known, the detection of explosives, drugs and other threats is one of the main security screening problems. To solve it, a $\mathrm{THz}$ radiation has been actively used during the past twenty years [1]-[18]. The THz TDS also provides new opportunities in the field of non-destructive testing [19], pharmaceutical control [20], in studying technologically-related materials ranging from nanostructures to strongly correlated electron materials [21, 22].

Currently, the standard THz TDS method of the substance detection is based on a comparison of absorption frequencies of a substance under consideration with standard absorption frequencies from the database. It should be stressed that under the standard method we mean not the ways of the $\mathrm{THz}$ signal measuring and corresponding experimental details, but only the fact that eventually the standard $\mathrm{THz}$ TDS deals with the spectral characteristics (Fourier spectra, absorbance or reflectance spectra) of the $\mathrm{THz}$ signal registered using those or other setups. Unfortunately, this method possesses obvious great disadvantages. For example, it was shown that many ordinary substances have the same absorption frequencies belonging to explosives. This leads to efficiency decreasing of the standard THz-TDS method using. Opaque packaging and complicated surface of the sample, and high humidity of an ambient medium also decrease this method efficiency [10]-[14]. However, it should be stressed that the THz-TDS method may be applied with high efficiency for substance investigation and database development under laboratory conditions.

In the present paper, we continue to demonstrate essential limitations of the standard THz-TDS method using for the de- tection and identification of substances under real conditions - at long distance of about $3.5 \mathrm{~m}$, in ambient air with relative humidity of about $50 \%$. Our investigations demonstrate zerovalue efficiency of this method in real conditions. For this purpose, we use a neutral substance - a chocolate bar. Another example is a semiconductor identification under laboratory conditions - at short distance of about $15 \mathrm{~cm}$, in dry air with relative humidity less than $2 \%$. We show that the standard $\mathrm{THz}-$ TDS method detects the spectral features of hazardous substances in the commonly used neutral materials both under real and laboratory conditions. It is important, that this property is not inherent to any particular equipment, for example, to that used in the Lomonosov Moscow State University, because all data, which we used, were from various laboratories.

Measurement of the $\mathrm{THz}$ signal reflected from the chocolate bar at long distance was made at Lomonosov Moscow State University (Moscow, Russia). The THz signal transmitted through the RDX sample was registered in the Center for Terahertz Research, Rensselaer Polytechnic Institute (NY, USA); transmitted $\mathrm{THz}$ signals MA, MDMA, n-Si, p-Si - at Capital Normal University (Beijing, China).

At first glance, the example with the semiconductor may seem strange - why do we search for explosive or sugar in Si-wafer? However, currently almost every person has some electronic device (cell phone, or tablet PC) that contains semiconductor elements. If the false detection of explosives or some neutral substance instead of cell phone will take place many times, the remote screening efficiency will tend to zero.

We believe that the main disadvantage of the standard $\mathrm{THz}-$ 
TDS method consists in the following: it is impossible to make a conclusion about the actual absence of hazardous substances in the neutral sample under consideration. This fact leads to zero-value probability of the device developing, which can detect dangerous substances with high probability, because it will lead to a large number of false alarms. On the other hand, approach for the detection and identification of substances can be based on the spectral dynamics analysis (SDA) method [23]-[26], which may be one of the ways to overcome the THz-TDS method disadvantages. In this method the timedependent spectral intensities are analyzed at the chosen frequencies and this allows us to avoid false alarms appearing and to detect the explosive absence in the neutral substances; moreover, detecting them. To illustrate that the method does not depend on the particular installation, we use the results of experiments performed at different times and in different laboratories: in Poland (Military University of Technology, Warsaw) and Lithuania (Semiconductor Physics Institute, Vilnius).

Earlier, the SDA-method was successfully used for the identification of neutral substances, explosives and drugs in the transmission and reflection mode [23]-[26], [40]. For quality assessment of a probability for dangerous substance presence in a substance under consideration, new integral criteria for the detection and identification were proposed for the first time in [27]. In [28] these criteria were applied for the PWM C4 explosive detection with a complicated surface in the reflection mode using data from two Universities mentioned above. These facts illustrate a weak sensitivity of the SDA-method to specific measurements. In [29]-[34], [40] the identification of substance, placed at long distances also was made.

Despite these investigations, there is a least one more problem that was not discussed at all: an influence of a diameter of the $\mathrm{THz}$ beam, which falls on a semiconductor wafer, on spectrum of the pulse transmitted through semiconductor. We show its essential influence on the spectrum in the frequency range $v>1.65 \mathrm{THz}$. The beam diameter is changed using an aperture placed in front of a sample.

\section{THE SETUP}

In our physical experiments, we exploit a $\mathrm{THz}$ spectrometer EKSPLA developed by Teravil Inc., Lithuania [32]. It uses a femtosecond fiber laser, which generates laser pulse with average power about $1 \mathrm{~W}$, with $1030 \mathrm{~nm}$ centre wavelength, $75 \mathrm{MHz}$ repetition rate, and its pulse duration is $80 \mathrm{fs}$. Lowtemperature grown $\mathrm{GaBiAs}$ is used as photoconductor. The spectral range of the spectrometer is $0.1-5.0 \mathrm{THz}$, signal-tonoise is better than $10^{3}: 1$ (at $\left.2 \mathrm{THz}\right), 10^{5}: 1$ (at $1 \mathrm{THz}$ ) and $10^{6}: 1$ (at $0.4 \mathrm{THz}$ ), spectral resolution is better than $10 \mathrm{GHz}$ (fast scan), $2.5 \mathrm{GHz}$ (combined mode). The schematic of the system can be found in http://www.ekspla.com. We use a parabolic mirror for the $\mathrm{THz}$ beam focusing on the object. Because the femtosecond fiber laser has average power of about $1 \mathrm{~W}$ and the laser beam splits many times, we use additional flat mirror behind the object. Therefore, our setup operates in reflection-transmission mode simultaneously for each measurement. The measurements were carried out under real con- ditions with a temperature $18^{\circ} \mathrm{C}$ and the relative humidity of about $50 \%$. The distance between the parabolic mirror and the object was about 3.5 meters.

Measurement of explosive RDX was made in transmission mode using the Teraview TPS 3000 unit in the standard configuration. The main spectrometer parameters are: spectral range is equal to $0.06-3.6 \mathrm{THz}$, signal-to-noise is better than $4000: 1$, dynamic range is higher than $3 \mathrm{OD}$ in the range: from $2 \mathrm{~cm}^{-1}$ to $100 \mathrm{~cm}^{-1}$, spectral resolution is equal to $0.06 \mathrm{THz}$ and rapid scan mode can do 30 scans/second [14, 26]. The distance between a sample and the spectrometer receiver did not exceed $30 \mathrm{~cm}$. Measurement procedure is described in detail in [9], but in our case, the RDX signal was measured in the ambient air

\section{SPECTRAL ANALYSIS}

\subsection{Spectral properties of chocolate}

In Figure 1(a), we present the sample investigated under real conditions, it is a chocolate bar with thickness $5-7 \mathrm{~mm}$. Let us notice again that the $\mathrm{THz}$ pulse falls on the sample and reflects from the flat metallic mirror placed behind the sample. Then, this reflected pulse passes the same distance toward the parabolic mirror of the $\mathrm{THz}$ spectrometer. Of course, a part of the $\mathrm{THz}$ pulse is reflected from various parts of the chocolate including its inhomogeneity.

Figure 1(b) shows the measured Chocolate $\mathrm{THz}$ signal on the time interval $t=[0,110]$ ps. The strong noise, which is observed in the signal, can be a result of the ambient medium action. Nevertheless, maximal amplitude of the THz signal is 4 times more than noise amplitude. Note that the frequency resolution depends obviously on the time interval, during which the measurements are performed. As a rule, its duration in the experiment with chocolate was approximately 100 ps. Consequently, the minimum frequency difference $\Delta v$, which can be resolved by computer processing, is $10 \mathrm{GHz}$. Let us remind, that the standard THz-TDS method deals with the spectrum of a $\mathrm{THz}$ signal main pulse. In our case, its duration is about 25 ps. With this aim, the Fourier spectrum (Figures 2(a) and 2(b)) and absorbance (Figures 2(c) and 2(d)) of the Chocolate main pulse are depicted in Figure 2 in the frequency ranges $v=[0,1.5] \mathrm{THz}$ (Figure 2(a)), [1.5, 3.2] THz (Figure 2(b)), where

$$
A=-\log _{10}\left(|P(v)| /\left|P_{R E F}(v)\right|\right) .
$$

$|P(v)|, \quad\left|P_{R E F}(v)\right|$ are the spectral amplitude modulus of the reference and Chocolate signal main pulse. The curve construction is made with the frequency resolution $\Delta v=0.01 \mathrm{THz}$. In order to provide such resolution, we have cut the main pulse out from the long measured signal on the time interval $t=[0,25] \mathrm{ps}$, and then extended the main pulse by zeros over the time $t=100 \mathrm{ps}$. As we usually use a Fourier spectrum (or spectral line dynamics) for the detection and identification of substance while in the most of the articles absorbance is used for these purposes, we stress that the spectrum minima (Figures 2(a) and 2(b)) are in a good agreement with maxima in absorbance (Figures 2(c) 


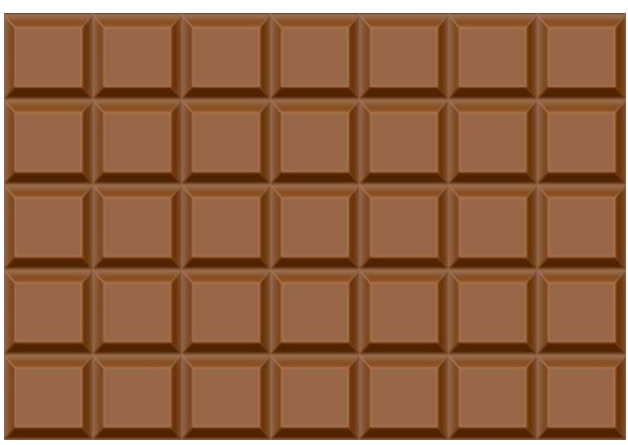

(a)

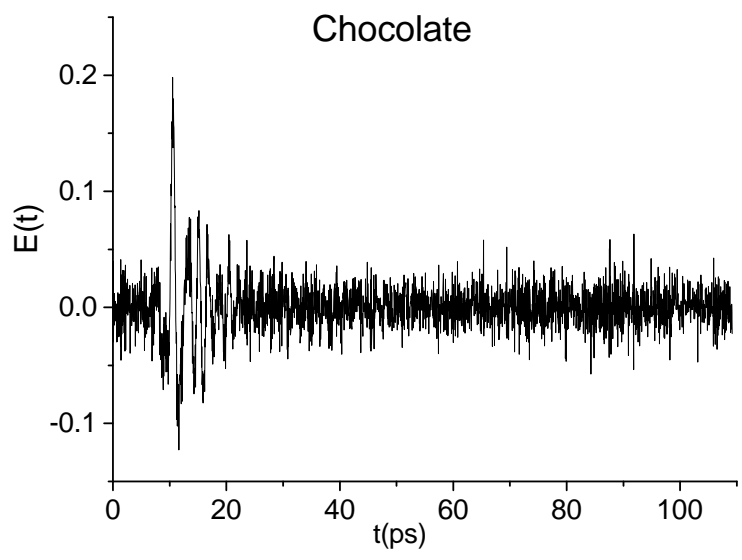

(b)

FIG. 1 Chocolate bar (a) and the Chocolate THz signal (b).

and 2(d)). Therefore, using of these spectral characteristics of the pulse (Fourier spectrum and absorbance) is equivalent for the detection problem. The Chocolate signal spectrum corresponding to the time interval $t=[0,110] \mathrm{ps}$ is depicted in [32].

It is obvious that in our case the chocolate does not contain dangerous substances. However, in the Fourier spectrum and absorbance (Figure 2) it is possible to see the spectral features of explosives and illicit drugs. Really, in accordance with $[6,7,9]$, the absorption frequencies of explosives are: $v=0.82,1.05,1.36,1.54,1.95,2.19,3.0 \mathrm{THz}$ for RDX; $v=1.78,2.51,2.82 \mathrm{THz}$ for HMX; $v=2.0,2.16,2.84 \mathrm{THz}$ for PETN. The absorption frequencies of drugs are $v=1.23,1.67,1.86 \mathrm{THz}$ (for MA) and $v=1.2,1.9 \mathrm{THz}$ (for MDMA) in the frequency range $v<2.5 \mathrm{THz}$ [36]. Let us remind that at studying the spectral properties of MA and MDMA [25, 32], the following absorption frequencies were found: $v=1.25,1.65,1.85,2.65 \mathrm{THz}$ for MA and $v=1.25,1.95 \mathrm{THz}$ for MDMA, which are in good agreement with [36] and they are used below in our investigations.

As usual, a substance is detected by using the set of known absorption frequencies from database. However, at measuring under real conditions, the part of such set of frequencies belonging to the measured pulse spectrum may be distorted by sample surface inhomogeneity or attenuated by water vapor (or other gases). So, one can find in the spectrum and absorbance the part (or only one) of these absorption frequencies. Therefore, we have to tell about belonging of the part (or only one frequency) to dangerous substance. That is why we show in Figure 2 (and also in Figures 3 and 4) not only the sets of absorption frequencies, but the single minima (single peaks in absorbance) too, for example, $v=1.94 \mathrm{THz}$ for MDMA in Figures 2(b), 2(d) and 2(f). In the spectrum (Figures 2(a) and 2(b)) and absorbance (Figures 2(c) and 2(d)) one can also see extremes $v=1.8 \mathrm{THz}$ and $v=1.7 \mathrm{THz}$, which are close to characteristic absorption frequencies of pure $\mathrm{Su}$ crose $v=1.83 \mathrm{THz}$ [12] and Chocolate $v=1.75 \mathrm{THz}$ [29]. Our aim is to distinguish these frequencies. In other words, we want to answer the question whether these minima (or peaks in absorbance) belong to dangerous substances or not. For such cases, we develop and improve currently our criteria and show that they allow us to distinguish and separate the false absorption frequencies.

The false absorption frequencies can appear in the spectrum for several reasons: the presence of noise, the inhomogeneous structure of the sample and its surface, the presence of impurities with similar absorption frequencies, the influence of the environment - for example, water vapor in the air, the presence of layered covering, etc. Additional ingredients containing in chocolate (aromatic and food additives) or some agents used during manufacturing may also induce the appearance of certain frequencies.

Obviously, a spectral resolution of a THz spectrometer influences on the detection and identification of a substance. In ideal conditions (without presence of water vapor and other features mentioned above), the increase in spectral resolution leads to the increase in identification probability, because we can use the fine structure of the $\mathrm{THz}$ spectrum. However, the noise presence in the $\mathrm{THz}$ pulse can cardinally change this situation. The reason is obvious. Small-scale perturbations modulate a $\mathrm{THz}$ pulse and, therefore, many additional extremes appear in the spectrum. Nevertheless, in our opinion, there is a simple approach, which can partly avoid and distinguish these frequencies from the absorption frequencies of dangerous substances. Taking into account a spectral width of the absorption frequency of the standard substance from the database, one can decrease the spectral resolution of calculated spectrum in order to remove the small-scale perturbation influence. Figure 2 illustrates this statement by comparing the spectra calculated with various resolution.

Figures 2(e) and 2(f) shows the main pulse Fourier spectrum calculated with spectral resolution $\Delta v=0.04 \mathrm{THz}$. We see decreasing the number of minima in comparison with the spectrum, calculated with spectral resolution $\Delta v=0.01 \mathrm{THz}$ (Figures 2(a) and 2(b)). In particular, we do not observe minima at frequencies close to RDX and MA absorption frequencies $v=0.82 \mathrm{THz}$ and $v=1.25 \mathrm{THz}$, correspondingly. In our opinion, the appearance of these minima in the spectrum (Figures 2(a) and 2(b) was caused by small-scale signal modulation due to external factors, or the sample structure, or the noise. It should be noted that other minima, which are close to absorbance frequencies of RDX, MA, MDMA as well as Sucrose and Chocolate, retain in the spectrum. The same situation takes place in the absorbance, obtained with spectral resolution $\Delta v=0.04 \mathrm{THz}$ (not shown in Figure 2). Taking into account this fact, in Section 5, we will deal with spectrum obtained with resolution $\Delta v=0.04 \mathrm{THz}$. As we see, the sample with chocolate demonstrates the spectral features of sugar, 


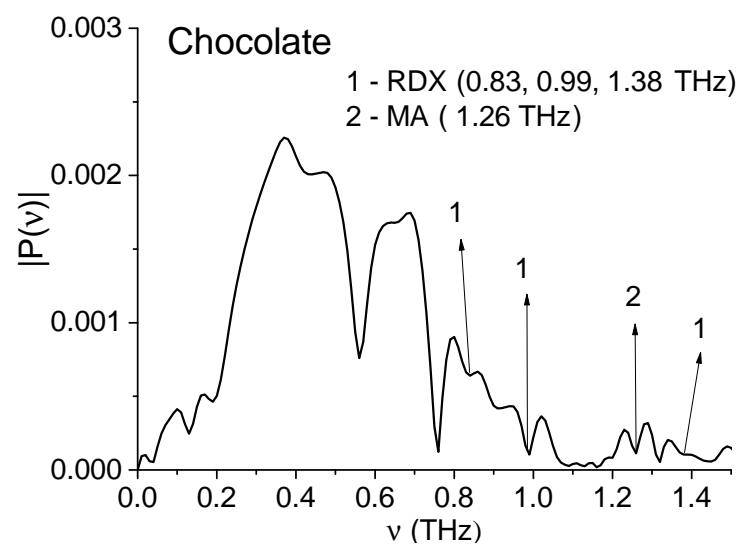

(a)

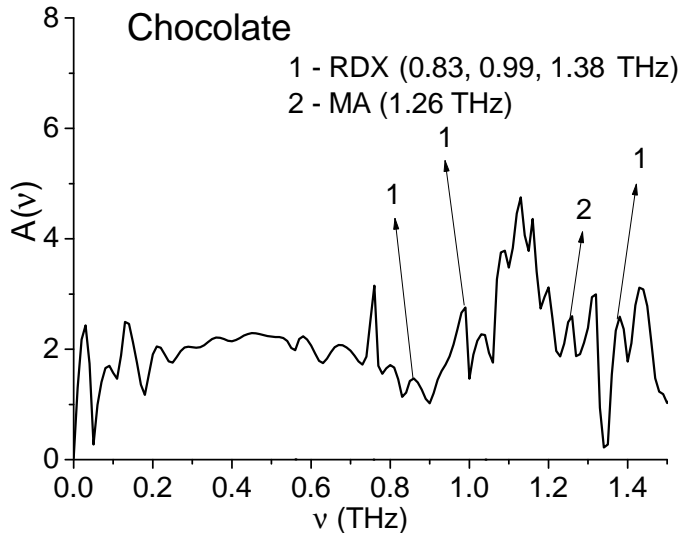

(c)

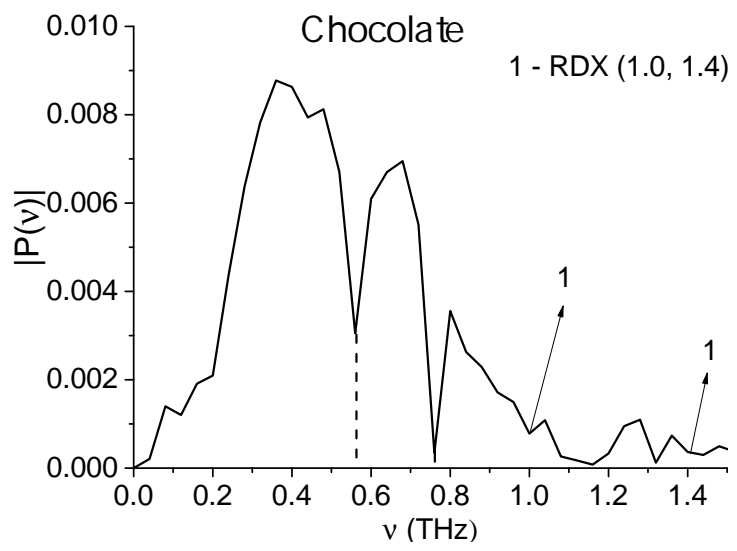

(e)

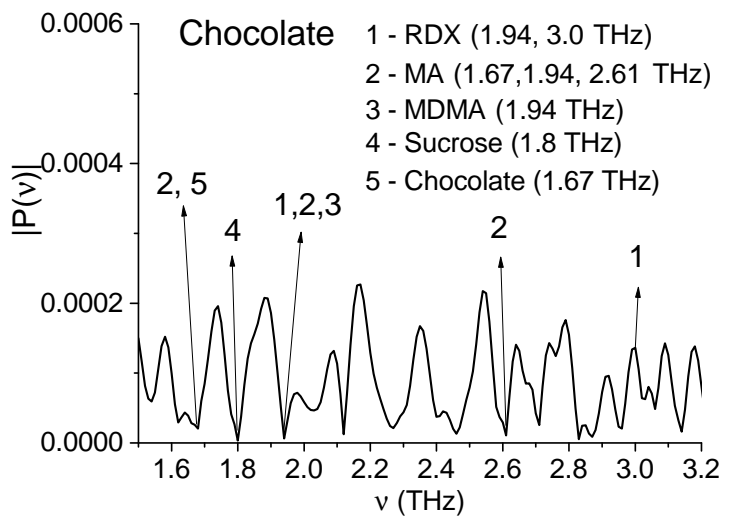

(b)

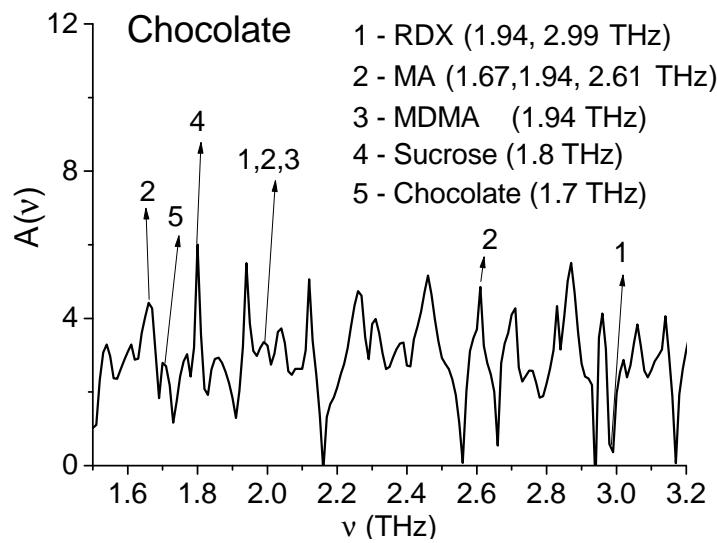

(d)

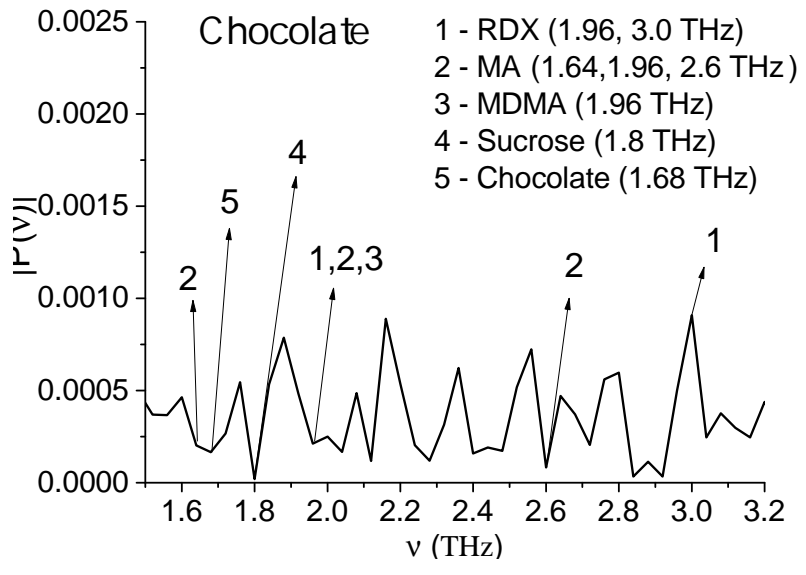

(f)

FIG. 2 Fourier spectrum (a), (b), (e), (f) and the absorbance (c), (d) of the Chocolate signal main pulse in the frequency ranges $\nu=[0,1.5]$ THz (a), (c), (e), and [1.5, 3.2] THz (b), (d), (f) calculated with spectral resolution $\Delta \nu=0.01 \mathrm{THz}(\mathrm{a})-(\mathrm{d}), 0.04 \mathrm{THz}(\mathrm{e}),(\mathrm{f})$.

chocolate, explosives and drugs for both spectral resolution $\Delta v=0.01 \mathrm{THz}$ and $0.04 \mathrm{THz}$.

\subsection{Spectral properties of Si-based semiconductors}

Below we analyze spectral properties of n-Si, p-Si semiconductors and silicon wafers with resistivity 35 and $40 \Omega \cdot \mathrm{cm}$ and measured with $12 \mathrm{~mm}$ aperture placed in front of the sample. We will call these signals as n-Si, p-Si, Si-35-12 and Si-40-12 for brevity. Our purpose is to show that even under laboratory conditions (at low humidity and at short distance from the receiver) the standard $\mathrm{THz}$ TDS method detects in the semiconductors the absorption frequencies, which belong to hazardous substances as well to neutral ones: L-tartaric acid (LTA) and sucrose. This means that we can detect any substance, which is really absent in the sample.

The n-Si, p-Si THz signal measurements were made at Capital Normal University (Beijing, China) by using a Ti:sapphire regenerative amplifier (Spectra-Physics Mai Tai) delivering optical pulses with a duration of $100 \mathrm{fs}$ and a central wavelength of $800 \mathrm{~nm}$ at a repetition rate of $1 \mathrm{kHz}$, with noise less than $0.15 \%[36,43]$. The transmitted $\mathrm{THz}$ signal was detected by free-space electro-optic sampling in a 1-mm-thick $<110>$ ZnTe crystal with the sampling pulse [37]. The path with $\mathrm{THz}$ radiation was purged by nitrogen to prevent absorption by atmospheric water vapor. The p-Si and n-Si samples were $0.5 \mathrm{~mm}$ thickness. 


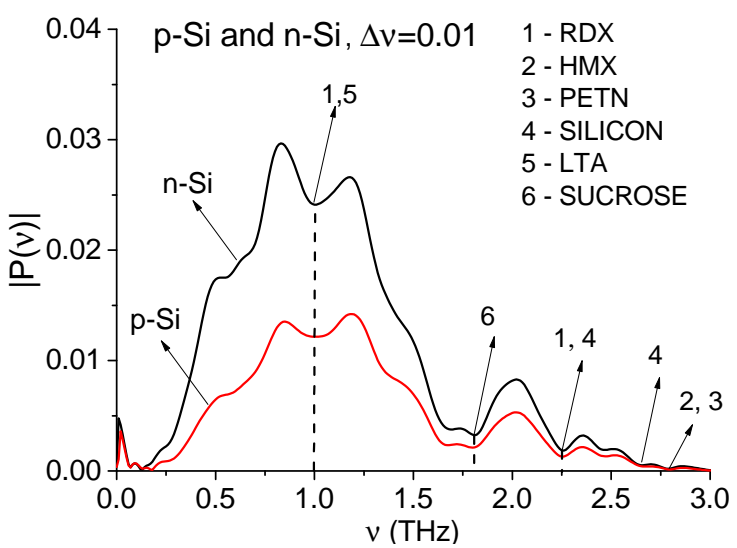

(a)

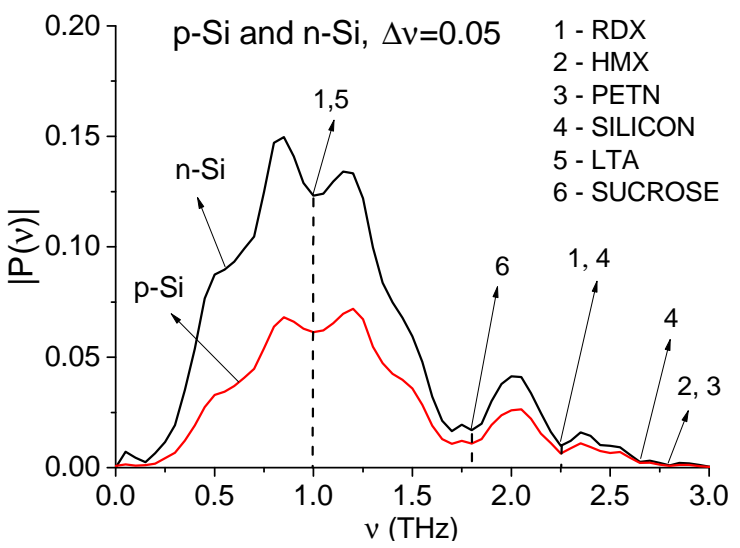

(c)

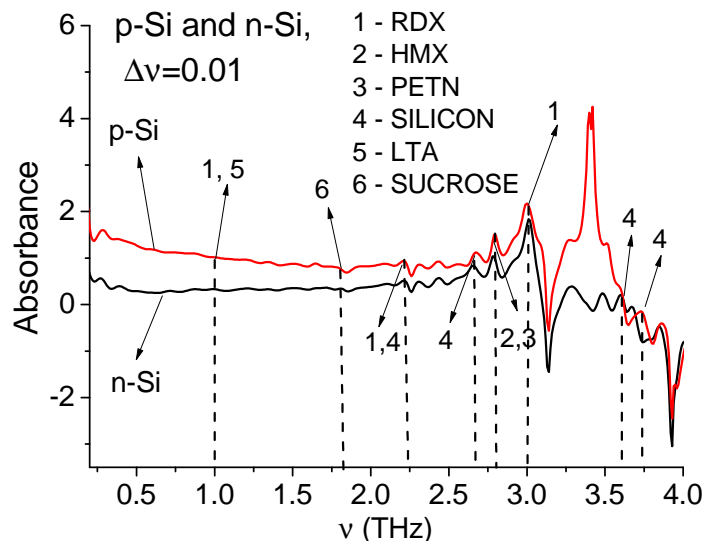

(e)

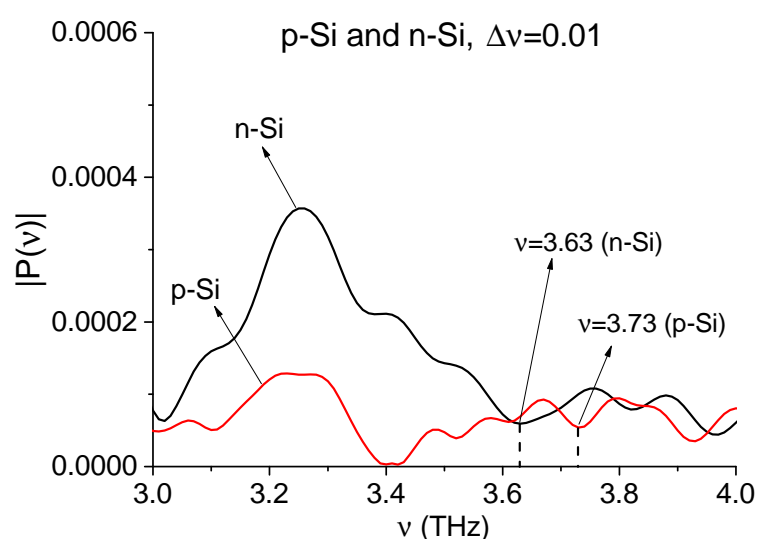

(b)

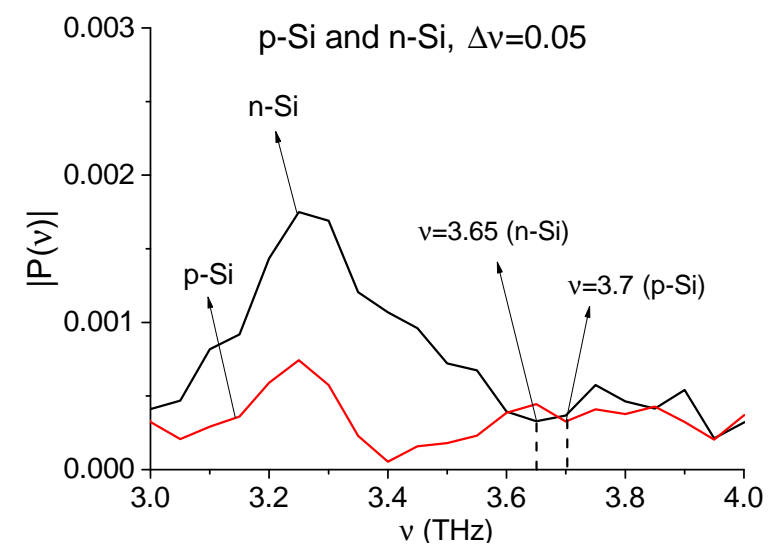

(d)

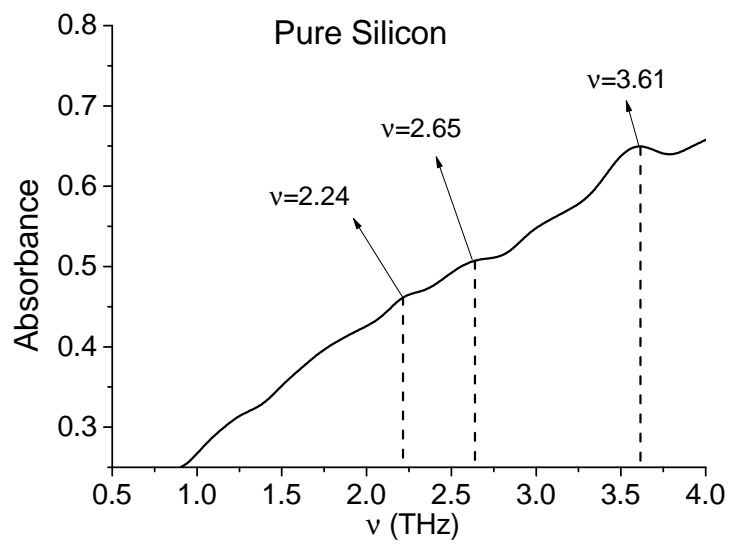

(f)

FIG. 3 The main pulse spectra of the $\mathrm{n}$-Si and p-Si signals in the frequency ranges $\nu=[0.0,3.0]$ THz (a), (c), [3.0, 4.0] THz (b), (d) calculated with the frequency resolution $\Delta \nu=0.01 \mathrm{THz}(\mathrm{a}),(\mathrm{b}),(\mathrm{e}), 0.05 \mathrm{THz}(\mathrm{c}),(\mathrm{d})$. Absorbance of $\mathrm{n}-\mathrm{Si}, \mathrm{p}-\mathrm{Si}$ (e) and monocrystalline silicon (f).

The Si-35-12 and Si-40-12 THz signals were measured at the South China Normal University (Guangzhou, China) in transmission mode, at short distance about $30 \mathrm{~cm}$ from the receiver at room temperature in the open air with non-zero humidity (about 30\%). Measurements were made by using a Ti:Sapphire femtosecond oscillator (Mira 900, Coherent) pumped with a solid-state laser (Verdi-5, Coherent). The width and repetition rate of the laser pulse are $130 \mathrm{fs}$ and $76 \mathrm{MHz}$. The wavelength of the femtosecond laser pulses was tunable from 760 to $840 \mathrm{~nm}$ [35]. All Verdi are specified with the lowest noise commercially available ( $<0.02 \%$ RMS) [44].

In Figure 3 the main pulse spectra for the n-Si and p-Si signals in the frequency ranges $v=[0.0,3.0] \mathrm{THz}$ (Figures 3(a) and $3(\mathrm{c})$ ) and $[3.0,4.0] \mathrm{THz}$ (Figures $3(\mathrm{~b})$ and $3(\mathrm{~d})$ ) are shown for two time intervals $T=100 \mathrm{ps}$ (Figures 3(a) and 3(b)) and 20 ps (Figures 3(c) and 3(d)). So, the frequency resolution is equal to $\Delta v=0.01 \mathrm{THz}$ in Figures 3(a) and 3(b) and $0.05 \mathrm{THz}$ in Figures 3(c) and 3(d), correspondingly. In accordance with our purpose, first we compare spectral features of n-Si and p-Si semiconductors and explosives. One can see clearly the spectrum minima in Figures 3(a) and 3(c) corresponding to the explosive RDX, HMX, PETN absorption frequencies [6, 7, 9]. The absorbance (Figure 3(e)) also contains maxima at these frequencies (here spectral resolution is $\Delta v=0.01 \mathrm{THz}$ ). Moreover, the n-Si and p-Si signal spectra Figures 3(a) and 3(c) and absorbance Figure 3(e) also contain extremes close to the absorption frequencies of LTA $v=1.1 \mathrm{THz}$ and pure Sucrose $v=1.83 \mathrm{THz}[12,29]$. Therefore, one can do false detection of explosives or neutral substances in the semiconductors. 
Obviously, the semiconductor samples n-Si and p-Si demonstrate the spectral features of pure silicon. Let us note that a pure monocrystalline silicon possesses absorption frequencies $v=2.24,2.65 \mathrm{THz}$ (Figure 3(f)), which coincide with the corresponding minima in Figures 3(a) and 3(c). Data in Figure 3(f) are taken from the $\mathrm{THz}$ database [42] (RIKEN, Sendai). The paper [39] reported about the frequency $v=3.6 \mathrm{THz}$ as the well-resolved absorption frequency of high-resistivity, floatzone silicon. The minima of $\mathrm{n}-\mathrm{Si}$ signal at $v=3.63 \mathrm{THz}$ in Figure 3(b) and $v=3.65 \mathrm{THz}$ in Figure 3(d) are close to this frequency, the corresponding minimum of p-Si signal is shifted to $v=3.73 \mathrm{THz}$ (Figure 3(b)), $v=3.7 \mathrm{THz}$ (Figure 3(d)).

Comparing the spectra calculated with various spectral resolution (Figure 3), we see that the influence of spectral resolution on them is much less for $\mathrm{THz}$ signals measured in laboratory conditions than for noisy $\mathrm{THz}$ signals, measured in real conditions (see Figure 2). With decreasing the spectral resolution from $\Delta v=0.01 \mathrm{THz}$ in Figures 3(a) and 3(b) to $\Delta v=0.05 \mathrm{THz}$ in Figures 3(c) and 3(d), the number of minima do not change and we can see the same minima. This fact confirms the conclusion made in Subsection 3.1. That is, for very noisy $\mathrm{THz}$ signal the spectral resolution increasing leads to manifestation of the small-scale perturbations caused by the influence of the external factors, or the sample structure, or the noise. At the same time, the spectral resolution decreasing allows to exclude from consideration the false absorption frequencies caused by small-scale modulation of the signal. For the $\mathrm{THz}$ signals measured in the laboratory conditions, the spectral resolution decreasing affects the spectrum much smaller because the influence of the environment medium is practically absent. In Section 5, we will use the spectra obtained with resolution $\Delta v=0.05 \mathrm{THz}$ (Figure 3(c)).

Let us analyze another semiconductor samples. In Figure 4 the main pulse Fourier spectra of the Si-35-12 (Figures 4(a) and $4(\mathrm{~b})$ ) and Si-40-12 (Figures 4(c) and 4(d)) signals are depicted in the frequency ranges $v=[0,1.5] \mathrm{THz}$ (Figures 4(a) and 4(c)); $[1.5,3.7] \mathrm{THz}$ (Figures 4(b) and 4(d)). Fourier spectra were calculated on the time interval $t=[4,24] \mathrm{ps}$ (duration $T=20 \mathrm{ps}$ ) with spectral resolution $\Delta v=0.05 \mathrm{THz}$. The measurement of these signals was carried out with a non-zero humidity and we showed above that in this case, the spectral resolution increasing may lead to manifestation of the signal small-scale modulations. Then, below we do not present and do not use spectra obtained with the spectral resolution $\Delta v=0.01 \mathrm{THz}$. Note that in (Figures 4(b) and 4(d)) one can see minima at frequencies, which are close to the absorption frequencies of pure Silicon $v=2.24,2.65,3.61(3.6) \mathrm{THz}[39,42]$. As the maxima of Si-35-12 and Si-40-12 signal absorbance coincide with minima of the corresponding Fourier spectra, so we do not reproduce these figures in the paper. Absorbance for Si-35-12 and Si-40-12 signals can be found in [40].

As above, the Si-35-12 and Si-40-12 samples also demonstrate spectral features of the explosives as well as neutral substances.

These examples demonstrate very important feature of the standard THz-TDS method: the method is not able to detect the hazardous and neutral substance absence in the sample under investigation if they are really missing.

\section{SDA-METHOD AND INTEGRAL CORRELATION CRITERIA}

\subsection{Main features of the spectral dynamics analysis method (the SDA-method)}

The medium response to the action of a few-cycle $\mathrm{THz}$ pulse is strongly non-stationary. Hence, the analysis of the spectral intensity time evolution (spectral dynamics) at the chosen frequencies allows us to get much more information about the substance under investigation. Obviously, the standard $\mathrm{THz}$ TDS method gives only information about the spectrum averaged over the signal registration time, because it does not take into account the time-dependent changes of spectral intensities. Nevertheless, as we mentioned above, the standard $\mathrm{THz}$ TDS method is quite effective if the measurement of $\mathrm{THz}$ signal is carried out in laboratory conditions without the water vapor influence. However, in real conditions, the measured Fourier spectra (and therefore the absorption or reflection coefficients) are often noisy and distorted due to the water vapor influence, the packaging material, the inhomogeneous sample surface, etc. To overcome this effect on the detection and identification of a substance, we propose to use the integral correlation criteria (ICC).

ICC allow us to provide an effective estimation of spectral features presence of substances from database in the analyzed signal. The correlation coefficients themselves cannot be used to assess the presence of the standard substance spectral features in the signal under investigation due to their strong oscillations at different time moments because of the medium response modulation. Summing the correlation coefficient values corresponding to different time moments, one can decrease the noise influence, because at summing, the random fluctuations of this coefficient compensate partially each other and the noise influence is also partially suppressed. On the other hand, we accumulate positive information. This procedure is similar to the commonly used algorithm for noise influence decreasing in the measured signal. Therefore, integral correlation criteria are the very important part of the SDAmethod for detecting and identifying substances, especially under adverse conditions.

To check the efficiency of the ICC for the frequency detection, a computer modeling of a $\mathrm{THz}$ pulse interaction with medium possessing the known absorption frequency was carried out in [41]. We placed the medium inside the disordered layered structure. As a result, many false absorption frequencies appear in the spectrum of both transmitted and reflected signals. We use ICC for the detection of the substance with known absorption frequency in such conditions and demonstrate a feasibility and effectiveness of the method using this example.

In order to get information about the characteristic absorption frequencies and relaxation times of excited energy levels of molecules, we calculate the spectral intensity dynamics for the measured signal by using the Fourier-Gabor method. Then, 


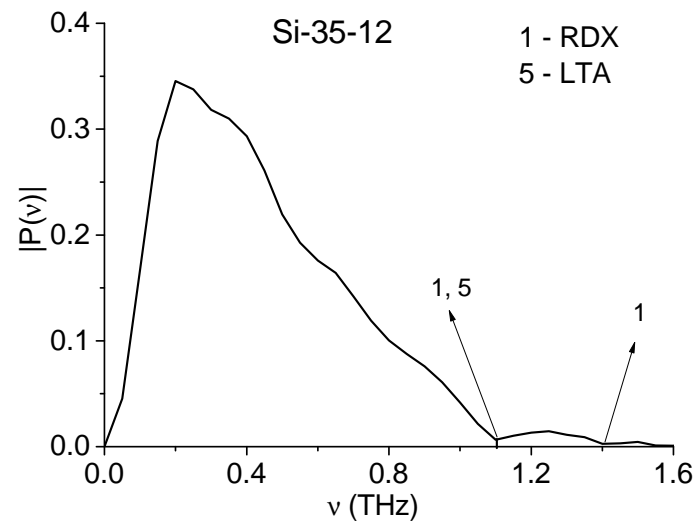

(a)

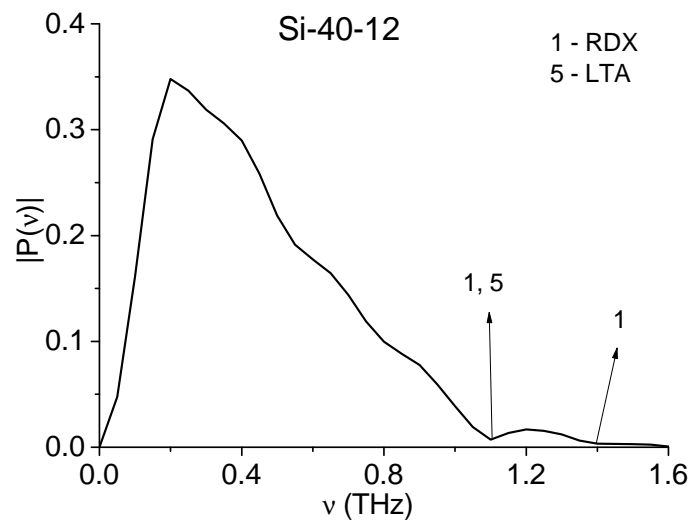

(c)

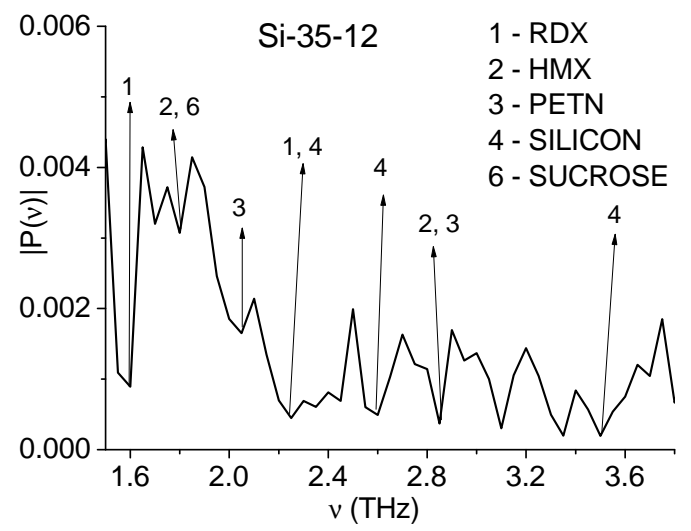

(b)

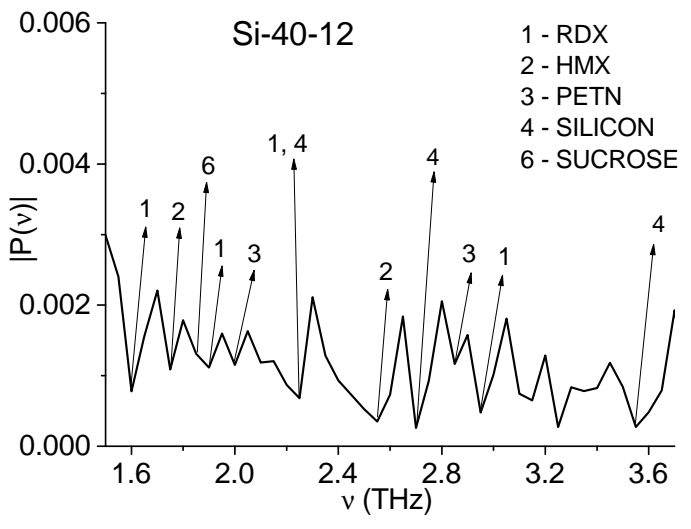

(d)

FIG. 4 Main pulse Fourier spectra of the Si-35-12 (a), (b) and Si-40-12 (c), (d) signals in the frequency ranges $\nu=[0,1.6]$ THz (a), (c), [1.5, 3.2] THz (b), (d) calculated with the frequency resolution $\Delta \nu=0.05 \mathrm{THz}$.

we move the spectral intensity (spectral line) dynamics of the standard $\mathrm{THz}$ signal at chosen absorption frequency along the spectral intensity dynamics of the analyzed signal during the chosen time interval. As a standard signal, we use the $\mathrm{THz}$ signal, transmitted through explosives, drugs and so on, and measured under laboratory conditions, or a signal measured in room conditions in view of water vapor absorption frequencies. Analyzing the integral correlation between these spectral dynamics, we can conclude about the presence or absence of the standard substance absorption frequencies in the sample. Moreover, it is possible to separate the frequencies corresponding to water vapor absorption frequencies despite their presence in the standard spectrum.

\subsection{Integral correlation criteria}

To calculate the integral correlation between the spectral line dynamics for the reflected or transmitted signal $S(t)$ and the standard transmitted signal $s(t)$ at chosen frequencies, we introduce the following notations. We denote the discrete set of spectral amplitude modulus for the standard transmitted signal at chosen frequency $v$ as $p_{v}=\left\{\left|p_{v}\left(t_{m}\right)\right|\right\}$, $m=1, \cdots, M_{1}$. Let us note, that the calculation of the spectral line (or spectral intensity) dynamics $P_{v}(t)$ at chosen frequency $v$ is described in a number of previous papers, for example, in [34]. The corresponding set of spectral amplitude modulus of the analyzed $\mathrm{THz}$ signal $S(t)$ at the frequency $v$ is denoted as $P_{v}=\left\{\left|P_{v}\left(t_{m}\right)\right|\right\}, m=1, \cdots, M_{2}$, and its part with $M_{1}$ components, which begins at time moment $t_{n}$, as
$P_{v}^{(n)}=\left\{\left|P_{v}^{(n)}\left(t_{n+m}\right)\right|\right\}$. Here $M_{1}$ and $M_{2}$ are the numbers of time moments, in which the spectral amplitudes are calculated. Both sets $p_{v}=\left\{\left|p_{v}\left(t_{m}\right)\right|\right\}$ and $P_{v}^{(n)}=\left\{\left|P_{v}^{(n)}\left(t_{n+m}\right)\right|\right\}$ must be averaged at each step $t_{n}$ to avoid the influence of their constant component on correlation value. Moving the set $p_{v_{1}}$ along the set $P_{v_{2}}$, we calculate in each time moment $t_{n}$ the correlation coefficient for two spectral dynamics

$$
\begin{gathered}
c_{p, P}\left(t_{n}\right)=\sum_{m=0}^{M_{1}-1} \frac{\left(\left|p_{v_{1}}\left(t_{m}\right)\right|-\overline{p_{v_{1}}}\right) \cdot\left(\left|P_{v_{2}}\left(t_{m+n}\right)\right|-\overline{P_{v_{2}}}\right)}{|| p_{v_{1}}-\overline{p_{v_{1}}}|| \cdot|| P_{v_{2}}^{(n)}-\overline{P_{v_{2}}}||}, \\
\overline{p_{v_{1}}}=\sum_{m=0}^{M_{1}-1} \frac{\left|p_{v_{1}}\left(t_{m}\right)\right|}{M_{1}}, \quad \overline{P_{v_{2}}}=\sum_{m=0}^{M_{1}-1} \frac{\left|P_{v_{2}}\left(t_{m+n}\right)\right|}{M_{1}} .
\end{gathered}
$$

Then, summing these correlation coefficients over time interval $\left[t_{0}, t_{n}\right]$, we get the following integral characteristic for the detection and identification problem [28]:

$$
C_{p, P}\left(t_{n}\right)=\sum_{m=0}^{n}\left|c_{p, P}\left(t_{m}\right)\right|, \quad n=0, \cdots, M_{2}-M_{1} .
$$

In the present paper, we use the modified criterion (Eq. (2)) taking into account the spectral brightness of each of frequencies $v_{1}$ and $v_{2}$ during the interval of correlation computing:

$$
C W_{p, P}\left(t_{n}\right)=\sum_{m=0}^{n}\left|c_{p, P}\left(t_{m}\right)\right| w_{1} w_{2}, \quad n=0, \cdots, M_{2}-M_{1}
$$

where $w_{1}=w\left(\left|P\left(v_{1}\right)\right|\right), w_{2}=w\left(\left|P\left(v_{2}\right)\right|\right)$ are the weight coefficients. Obviously, if $w_{1}=1$ and $w_{2}=1$, we get integral characteristic (2). 


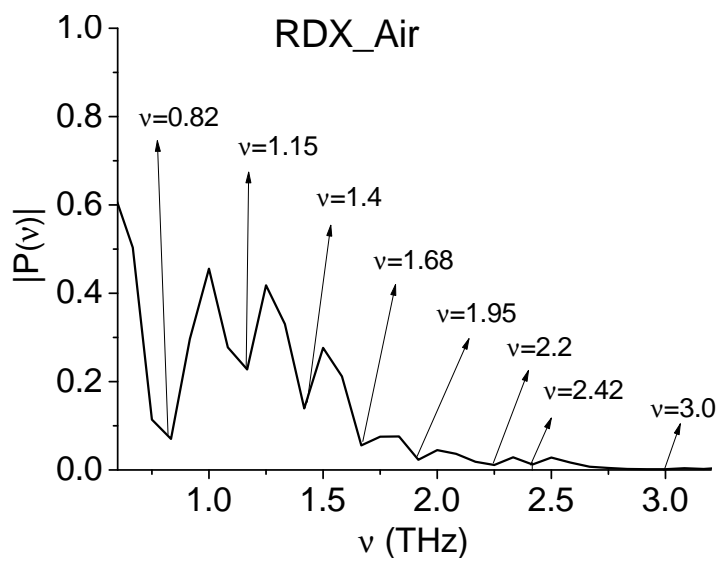

(a)

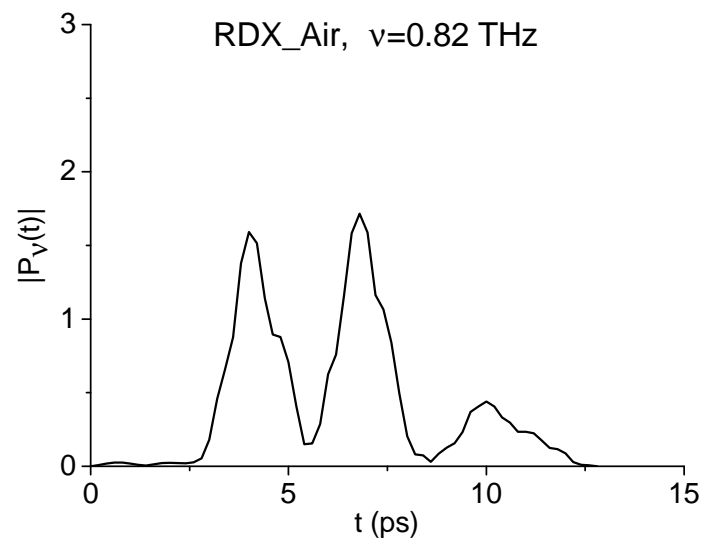

(c)

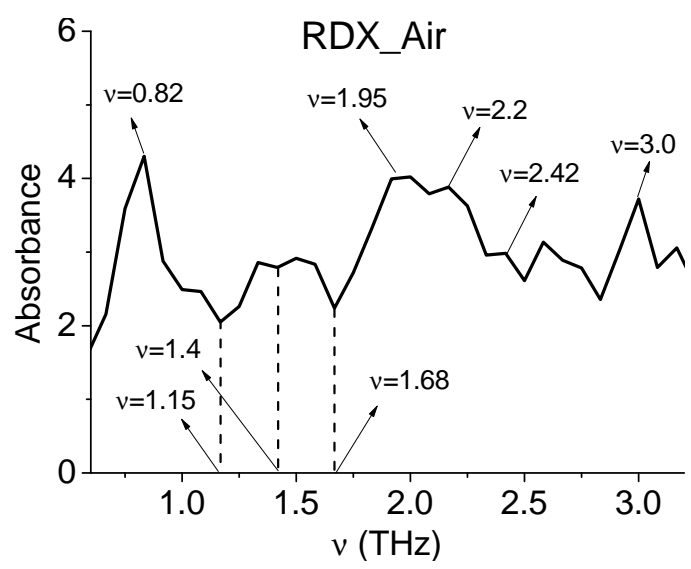

(b)

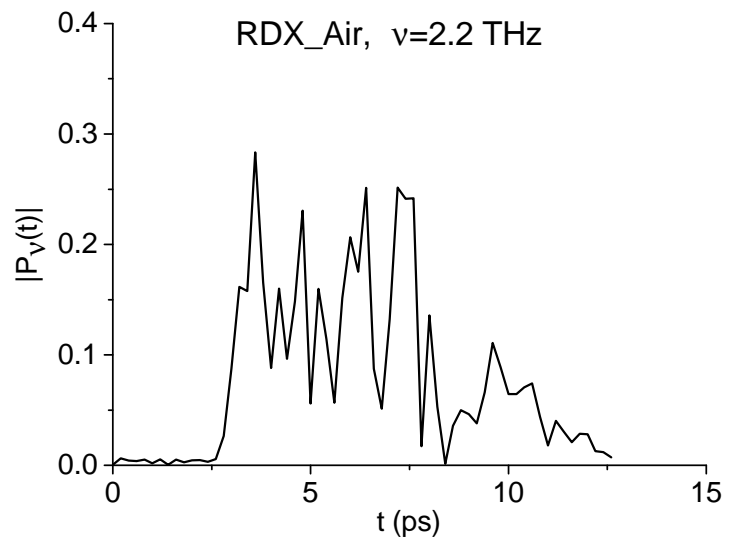

(d)

FIG. 5 Fourier spectrum of the standard RDX_Air signal (a) and absorbance (b) in the frequency range $\nu=[0.6,3.2]$ THz and spectral line dynamics of the RDX_Air signal at $\nu=0.82(\mathrm{c}), 2.2(\mathrm{~d}) \mathrm{THz}$.

Along with the criterion (3) we consider another criterion, in which the sets $p_{v_{1}}^{2}=\left\{\left|p_{v_{1}}\left(t_{m}\right)\right|^{2}\right\}, P_{v_{2}}^{2}=\left\{\left|P_{v_{2}}\left(t_{m}\right)\right|^{2}\right\}$ will be used instead of the sets $p_{v_{1}}$ and $P_{v_{2}}$ :

$$
\begin{gathered}
C W_{p, P}^{S Q}\left(t_{n}\right)=\sum_{m=0}^{n}\left|C_{p^{2}, P^{2}}\left(t_{m}\right)\right| w_{1}^{2} w_{2}^{2}, \\
n=0, \cdots, M_{2}-M_{1} .
\end{gathered}
$$

In certain cases, it is necessary to use one more criterion, which was introduced in [28] and allows us to assess the similarity (or likeness) of two spectral line dynamics

$$
L_{p, P}\left(t_{n}\right)=\sum_{m=0}^{n} l_{p, P}\left(t_{m}\right), \quad n=0, \cdots, M_{2}-M_{1}
$$

where

$$
\begin{aligned}
& l_{p, P}\left(t_{n}\right)=1-\frac{\left\|\left(p_{v_{1}}-\overline{p_{v_{1}}}\right)_{N}-\left(P_{v_{2}}^{(n)}-\overline{P_{v_{2}}}\right)_{N}\right\|}{\left\|\left(p_{v_{1}}-\overline{p_{v_{1}}}\right)_{N}\right\|+\left\|\left(P_{v_{2}}^{(n)}-\overline{P_{v_{2}}}\right)_{N}\right\|}, \\
& n=0, \cdots, M_{2}-M_{1} .
\end{aligned}
$$

The subscript $N$ indicates that the corresponding variable in Eq. (6) is normalized, for example, in $L_{2}$ norm.

\section{IDENTIFICATION OF CHOCOLATE AND SEMICONDUCTORS}

\subsection{The RDX absence detection in the chocolate sample}

In order to show the explosive RDX absence in the Chocolate sample, we will use the THz signal transmitted through the tablet containing 10\% RDX and 90\% PE in the ambient air as a standard one (we call it as RDX_Air signal). The measurement was performed during the short time interval $0<t<10 \mathrm{ps}$ at the room temperature $22^{\circ} \mathrm{C}$ and relative humidity of about $50 \%$. Since the measured $\mathrm{THz}$ pulse shape is shown in [28], then its Fourier spectrum (5(a)) and absorbance (5(b)) only are depicted in Figure 5 in the frequency range $v=[0.6,3.2] \mathrm{THz}$. In [28] we showed that the minima at frequencies $v=1.15,1.4,1.68 \mathrm{THz}$ in (a) are caused by the strong water vapor absorption of the $\mathrm{THz}$ radiation. However, the minima at $v=0.82,1.95,2.2,2.42,3.0 \mathrm{THz}$ (a) coincide with the absorbance maxima (b), and they can be used for the RDX identification, because they are in a good agreement with the RDX absorption frequencies given in other papers $[6,7,9]$.

As we mentioned above in Section 3.1, the part of the set of known absorption frequencies can be distorted while measuring under real conditions. That is, the absence of the minima at these frequencies in the spectrum of the signal under investigation does not mean the absence of the substance itself and it is necessary to analyze also such frequencies. The Chocolate 


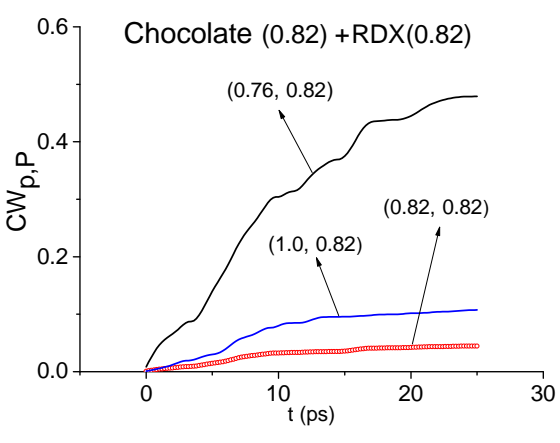

(a)

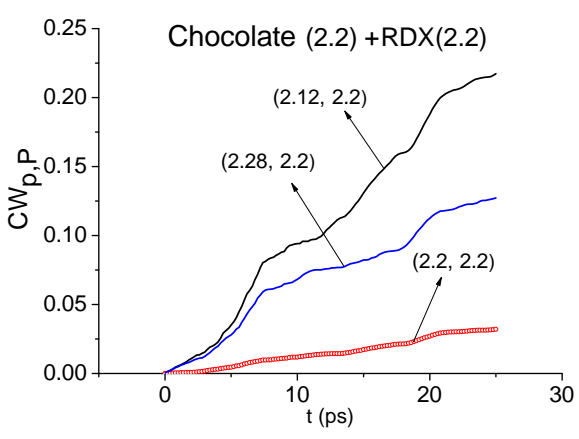

(b)

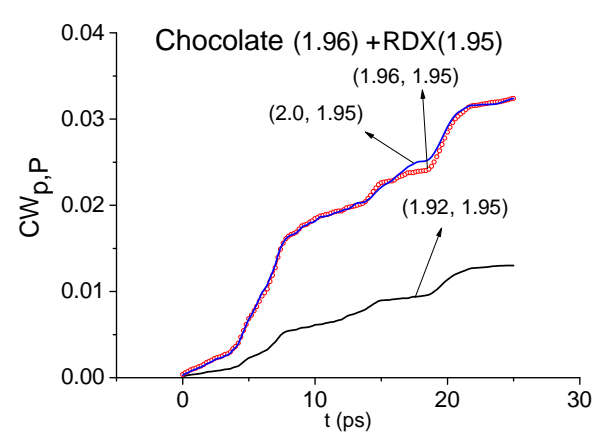

(c)

FIG. 6 Time-dependent integral criterion $C W_{p, p}\left(t_{n}\right)$ at the frequencies $\nu=0.82(\mathrm{a}), 2.2(\mathrm{~b}), 1.96$ (c) THz for the Chocolate signal and RDX_Air signal as a standard one.

signal spectrum (Figures 2(e) and 2(f)) does not contain minima at frequencies, which are equal to the RDX_Air absorption frequencies $v=0.82,2.2 \mathrm{THz}$. We will use the spectral line dynamics at these frequencies (presented in Figures 5(c) and 5(d)) for RDX detection in the Chocolate signal.

It should be stressed that the frequency $v_{1}$, belonging to a standard substance, is detected in the signal under investigation at the frequency $v$ if the integral criterion, calculated for the frequency pair $\left(v, v_{1}\right)$, lies above all other characteristics in the frequency detection range (FDR). As a rule, the boundaries of the FDR are the spectrum extremes closest to the analyzed frequency. And vice versa, the frequency $v_{1}$ is not detected if, at least, one of other lines lies above the integral criterion, calculated for the pair $\left(v, v_{1}\right)$, in this frequency range.

In Figure 6 the integral criterion $C W_{p, P}\left(t_{n}\right)$ evolution is shown at the frequencies $v=0.82$ and $2.2 \mathrm{THz}$ (Figures 6(a) and 6(b)), during the main pulse. In both cases, these RDX absorption frequencies are not detected in the Chocolate signal and, therefore, spectral features of RDX are absent in the signal under investigations.

It is very important for practice that the integral criteria in Eqs. (2) and (5) allow to show the explosive absence even if the spectrum of the $\mathrm{THz}$ signal under investigation contains the minimum, which is close or equal to the absorption frequency of the standard substance. To illustrate this, we consider the absortpion frequency $v=1.96 \mathrm{THz}$ of the Chocolate signal (Figure 2(f)), which is close to the RDX absorption frequency $v=1.95 \mathrm{THz}$. Figure 6(c) shows the integral criterion $C W_{p, P}\left(t_{n}\right)$ evolution, calculated for the frequency pair $v=(1.96,1.95) \mathrm{THz}$. We see that the criterion evolution line calculated for a pair $v=(2.0,1.95) \mathrm{THz}$ lies above the line, corresponding to the pair $v=(1.96,1.95) \mathrm{THz}$. So, the frequency $v=1.96 \mathrm{THz}$ is also not detected as the RDX absorption frequency in the Chocolate signal. Thus, using these three frequencuies, we show the RDX absence in the sample with chocolate. In the same way, it is possible to show the absence of illicit drugs MA, MDMA in this sample.

We stress that the integral criterion $C W_{p, P}^{S Q}\left(t_{n}\right)$ gives the same result as $C W_{p, P}\left(t_{n}\right)$, but it increases the detection contrast. This criterion is reasonable for using if the criterion $C W_{p, P}\left(t_{n}\right)$ gives close or coinciding lines for different frequency pairs [29].

\subsection{Sugar and chocolate detection in the sample}

Below we detect absorption frequencies of sugar and chocolate in the Chocolate signal. For this purpose, we use the standard transmitted THz signals Sucr10 and Choc10. The Choc10 signal was measured in the Institute for Spectroscopy RAS (Troitsk, Russia) at short distance of about $15 \mathrm{~cm}$ from $\mathrm{THz}$ receiver, at room temperature and with humidity of about $50 \%$. Measurements of transmitted Sucr10 THz signal was made in Semiconductor Physics Institute, Vilnius, Lithuania. The $\mathrm{THz}$ pulse shape and the corresponding Fourier spectra of the signals were depicted in $[29,31]$.

The Chocolate main pulse spectrum (Figures 2(e) and 2(f)) contains minima at the frequencies $v=1.7,1.8 \mathrm{THz}$, which are close to the absorption frequencies $v=1.75,1.85 \mathrm{THz}$ of the standard signals Choc10 and Sucr10, correspondingly. As we have seen above, the presence of a minimum at the standard substance absorption frequency in the spectrum of the signal under investigation does not mean the presence of this substance in the sample in reality. So, to find spectral properties of sugar and chocolate, we use the standard spectral line dynamics at frequencies $v=1.75,1.85 \mathrm{THz}$.

In Figure 7 the integral criterion $C W_{p, P}\left(t_{n}\right)$ evolution is depicted at frequencies $v=1.7 \mathrm{THz}$ (Figure 7(a)) and $v=1.8 \mathrm{THz}$ (Figure $7(\mathrm{~b})$ ) for the FDR $v=[1.6,1.72] \mathrm{THz}$ and $[1.72,1.88] \mathrm{THz}$, correspondingly. In both cases, these frequencies are detected as the absorption frequencies of Chocolate signal. We see that sugar and chocolate are found in the chocolate bar.

\subsection{Explosive and neutral substances absence in semiconductors}

In [33] we showed the RDX, HMX and PETN absence in the $\mathrm{n}$-Si and p-Si semiconductors with the help of integral correlation criteria in Eqs. (3)-(5). In the same manner, it is possible to show the absence of LTA and Sucrose in these samples (in Figure 3 we see the minima at frequencies, which are close to absorption frequencies of LTA and Sucrose). For this purpose, along with the standard Sucr10 $\mathrm{THz}$ signal, we will use the transmitted LTA10 THz signal. It was also measured in Semiconductor Physics Institute, Vilnius, Lithuania. The THz pulse shape and the corresponding Fourier spectrum of the signal can be found in [29,31]. 


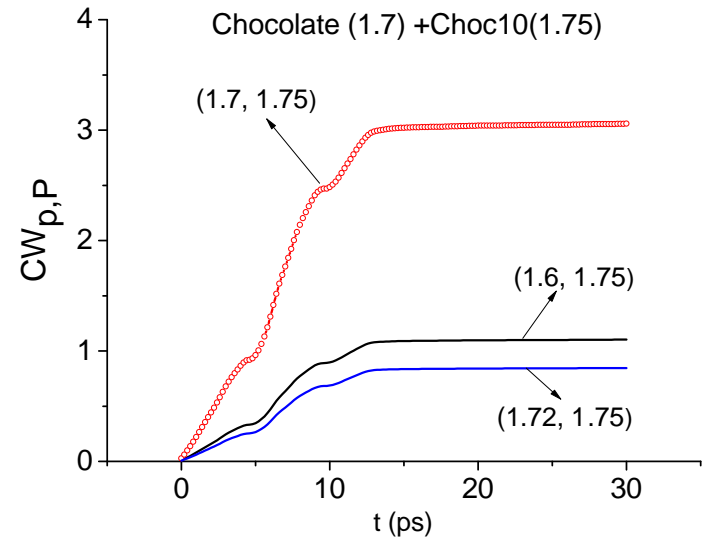

(a)

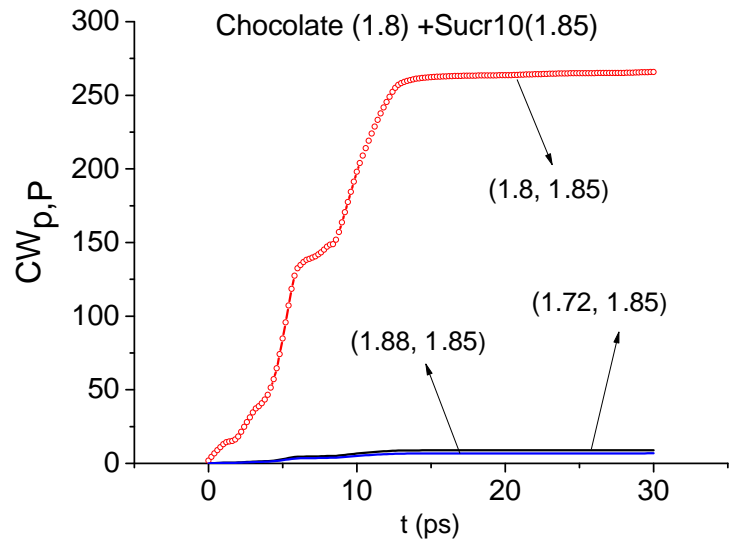

(b)

FIG. 7 Time-dependent integral criterion $C W_{p, P}\left(t_{n}\right)$ detecting the frequencies $\nu=1.7 \mathrm{THz}(\mathrm{a}), 1.8 \mathrm{THz}$ (b) for Chocolate signal and Choc10 (a), Sucr10 (b) as standard signals.

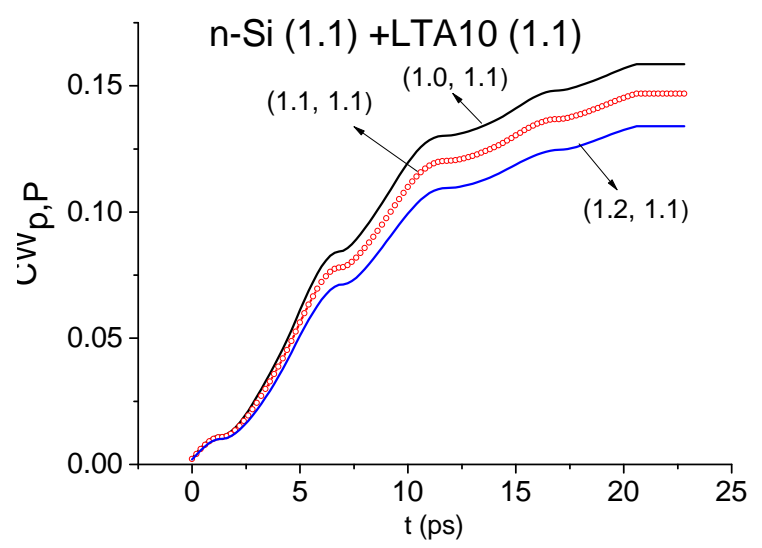

(a)

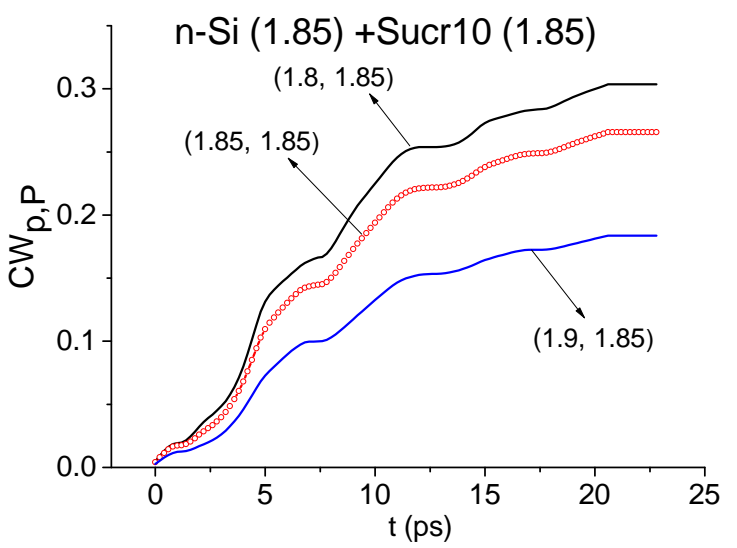

(b)

FIG. 8 Time-dependent integral criterion $C W_{p, P}\left(t_{n}\right)$ at frequencies $\nu=1.1 \mathrm{THz}(\mathrm{a}), 1.85 \mathrm{THz}$ (b) for $\mathrm{n}$-Si signal with LTA10 signal (a) and Sucr10 signal (b) as the standard ones.

In Figures 8(a) and 8(b) the integral criterion $C W_{p, P}\left(t_{n}\right)$ is presented for $v=1.1 \mathrm{THz}$ (Figure 8(a)), $1.85 \mathrm{THz}$ (Figure 8(b)), which are the absorption frequencies of the LTA10 and Sucr10 signals, correspondingly.

In both cases there is a line, which lies above the lines computed for the frequency pairs $v=(1.1,1,1) \mathrm{THz}$ (Figure 8(a)) and $v=(1.85,1.85) \mathrm{THz}$ (Figure $8(\mathrm{~b}))$, so, these frequencies are not detected as absorption frequencies of LTA10 and Sucr10 signals in the n-Si signal. Therefore, L-Tartaric Acid and Sucrose are absent in the n-Si sample. In the same way, one can demonstrate the LTA and Sucrose absence in $\mathrm{p}-\mathrm{Si}$ semiconductor.

In Figure 9 the evolutions of $C W_{p, P}\left(t_{n}\right)$ are shown for the Si35-12 signal also computed for the frequencies $v=1.1 \mathrm{THz}$ (Figure 9(a)), $1.85 \mathrm{THz}$ (Figure 9(b)) with LTA10 signal (Figure 9(a)) and Sucr10 signal (Figure 9(b)) as the standard ones. In Figure 9(a) one can see that the line corresponding to the pair $v=(1.1,1.1) \mathrm{THz}$ lies above others in the FDR $v=[1.0,1.2] \mathrm{THz}$. It means that the spectral line dynamics at frequency $v=1.1 \mathrm{THz}$ in the signals Si-35-12 and LTA10 have the same type. Nevertheless, the detection of the frequency $v=1.1 \mathrm{THz}$ in Figure 9(a) does not mean the presence of L-Tartaric Acid in the Si-35-12 sample, because it possible that some energy level transitions have the same relaxation times for different substances. Therefore, it is necessary to compare other spectral line dynamics. We see that the fre- quency $v=1.85 \mathrm{THz}$ in Figure 9(b) is not detected in Si-35-12 signal in the FDR $v=[1.8,1.9] \mathrm{THz}$ as well as the frequency $v=1.7 \mathrm{THz}$ (Figure 9(c)), which is the absorption frequency of LTA $[21,23]$, is not detected in the FDR $v=[1.6,1.8] \mathrm{THz}$. Therefore, L-Tartaric Acid and Sucrose substances are absent in the sample with Si-35-12 semiconductor. The same result is valid for Si-40-12 sample.

\subsection{Aperture influence on the semiconductors spectral properties}

Obviously, the detection and identification efficiency depends on a quality of the standard signal from the database. Among many factors influencing on the signal quality, the $\mathrm{THz}$ beam diameter can change the response of the medium under investigation. Consequently, a standard signal spectrum and its spectral line dynamics may also change, and the detection result can change as well. Therefore, understanding how the $\mathrm{THz}$ beam diameter influences on a measured signal, is a key question. To answer this question, below we compare the spectra of the THz signals passed through two types of semiconductors, with and without presence of aperture, placed in front of the sample. Our aim is to pay attention to this problem and to demonstrate its importance. We will call the $\mathrm{THz}$ signals measured without aperture as Si-35 and Si-40 signals, correspondingly.

In Figure 10 the $\mathrm{THz}$ signals Si-35-12 (10(a) and 10(b)) and 


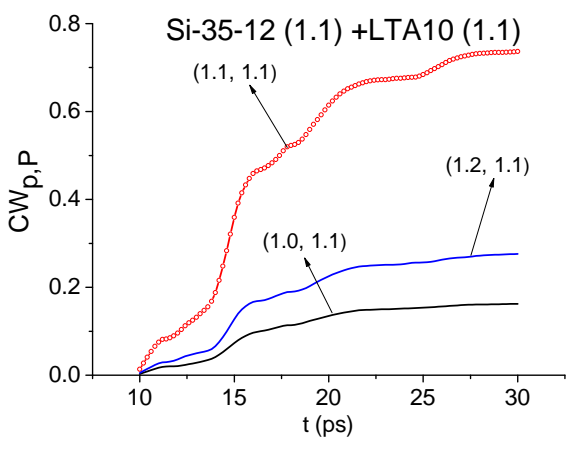

(a)

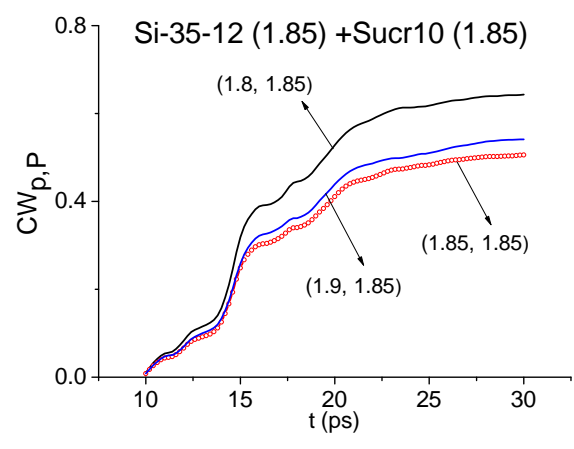

(b)

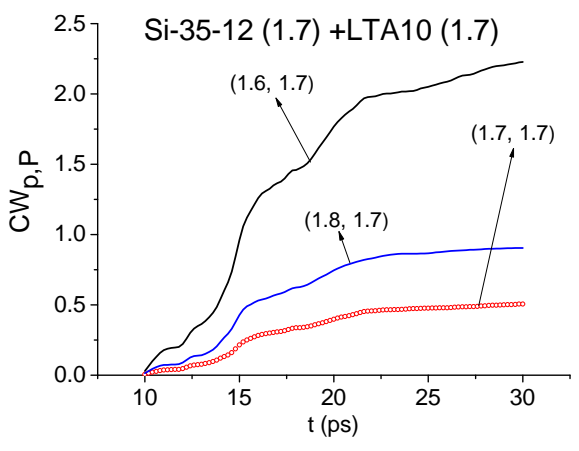

(c)

FIG. 9 Time-dependent integral criterion $C W_{p, P}\left(t_{n}\right)$ calculated for the analyzed frequencies $\nu=1.1 \mathrm{THz}(\mathrm{a}), 1.85 \mathrm{THz}(\mathrm{b}), 1.7 \mathrm{THz}(\mathrm{c})$ for Si-35-12 signal and LTA10 signal(a), (c), Sucrio signal (b) as the standard ones.

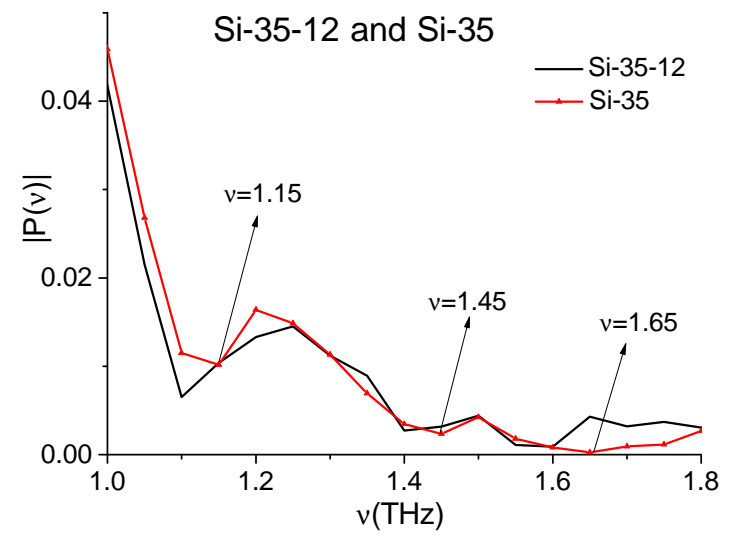

(a)

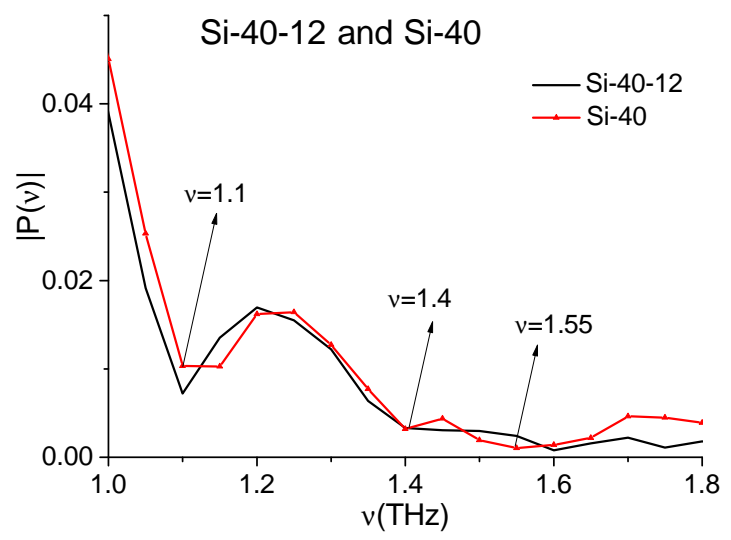

(c)

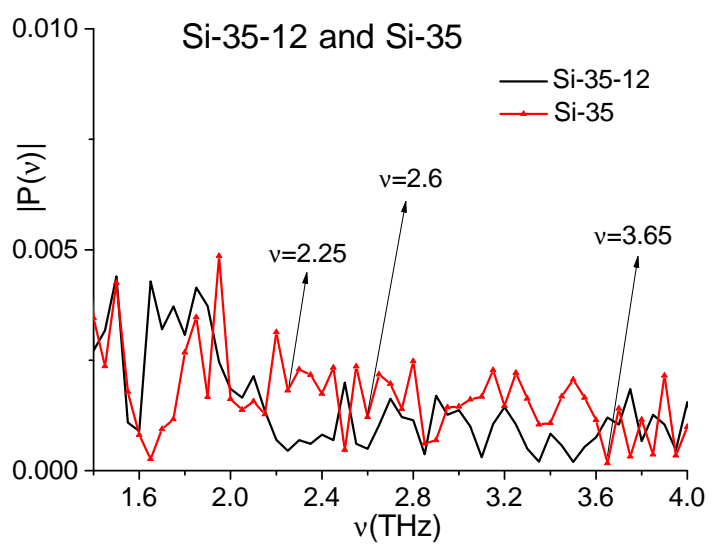

(b)

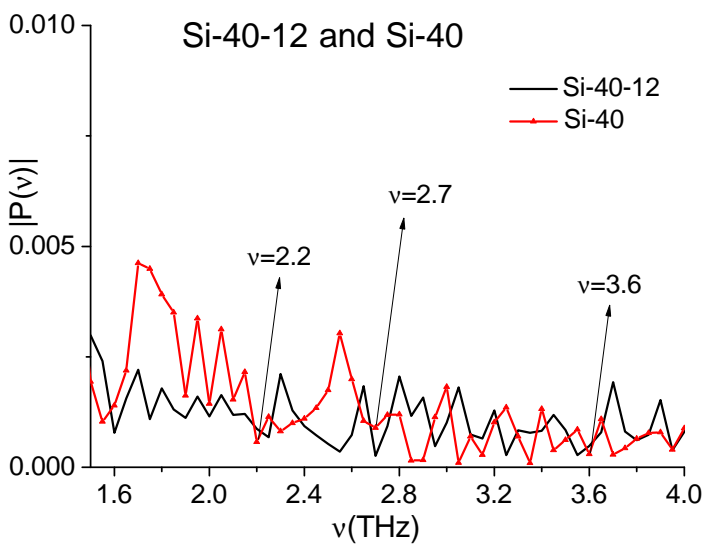

(d)

FIG. 10 Fourier spectra of the Si-35-12, Si-35 (a), (b) and Si-40-12, Si-40 (c), (d) THz signals in the frequency ranges $\nu=[1.0,1.8]$ THz (a), (c), [1.5, 4.0] THz (b), (d) calculated with the spectral resolution $\Delta v=0.05 \mathrm{THz}$.

Si-40-12 (10(c) and 10(d)) spectra are presented together with the corresponding $\mathrm{Si}-35$ and $\mathrm{Si}-40$ signals spectra. We see that these spectra, measured with and without $12 \mathrm{~mm}$ aperture, are similar for frequencies less than $1.6 \mathrm{THz}$ (Figures 10(a) and $10(\mathrm{c})$ ). A slight shift of minima only takes place for the spectra measured without aperture in this frequency range. As well, one can observe the small increasing of minima at frequency $v=1.1 \mathrm{THz}$ (Figures 10(a) and 10(c)) corresponding to the signals measured without aperture. It may be caused by the absorption frequency shifting for this case. One can see also other differences in spectra in the frequency range $v=[1.4,1.6] \mathrm{THz}$.
At the same time, the Si-35 and Si-40 signals spectra essentially differ from Si-35-12 and Si-40-12 signals spectra in the frequency range greater than $1.65 \mathrm{THz}$. We see that the positions and amplitudes of several extremes have dramatically changed in Figures 10(b) and 10(d). This fact should be taken into account for finding of spectral properties mismatching between semiconductor and dangerous and neutral substances. Let us note that the values of the Si-35 and Si-40 signals spectra minima corresponding to the absorption frequencies of pure Silicon $v=2.24,2.65,3.61(3.6) \mathrm{THz}[35,38]$, vary slightly in comparison with the corsponding values in Figure 4. Therefore, Si-35 and Si-40 THz signals measured 
without aperture, also demonstrate spectral feature of pure Silicon. However, an aperture presence can lead to appearance of various extremes in the signals spectra, which are shifted when changing the aperture diameter.

\section{CONCLUSIONS}

We have shown that the standard THz TDS method detects the spectral features of explosives RDX, HMX, PETN and illicit drugs MA, MDMA in the chocolate bar as well as explosives RDX, HMX, PETN and neutral substances L-tartaric acid and sucrose in silicon-based semiconductors despite their actual absence in the samples. This facts demonstrate the standard THz-TDS method non-efficiency not only under real conditions (at long distance, high relative humidity, influence of noise), but also under laboratory conditions (at short distance and low humidity). Therefore, this feature leads to a large number of false detections at security screening.

At the same time, the SDA-method together with the integral correlation criteria allow us to detect the dangerous and neutral substance absence in the samples. We demonstrate that these criteria allow to show the absence of RDX even if the spectrum of the analyzed $\mathrm{THz}$ signal contains the minimum that is close or equal to the absorption frequency of the standard signal. In order to enhance the detection reliability it is necessary to use the different types of integral criteria simultaneously.

We showed that the Chocolate signal demonstrates spectral features similar to those of sugar and chocolate, and the silicon-based semiconductor samples demonstrate similar spectral features of pure silicon. The influence of aperture on spectral properties of silicon wafers with different resistivity is also investigated. Thus, the aperture using leads to small decreasing of minimum at frequency $v=1.1 \mathrm{THz}$ and a slight shifting of other minima in the frequency range $v<1.65 \mathrm{THz}$. At the same time, the spectra of the signals measured with and without aperture, essentially differ in the frequency range $v>1.65 \mathrm{THz}$.

It is also shown that the increase in spectral resolution for very noisy $\mathrm{THz}$ signal leads to manifestating the small-scale perturbations caused by the influence of the external factors or the sample structure. At the same time, spectral resolution decreasing allows to exclude from consideration the false absorption frequencies caused by small-scale modulation. For $\mathrm{THz}$ signals measured in the laboratory conditions, the spectral resolution decreasing affects the spectrum much smaller.

Thus, the discussed method is promising and competitive tool for the effective detection and identification of various substances both in real and laboratory conditions in comparison with the THz TDS method, based on comparison of the substance spectra. The method can be used with success for security screening, non-destructive testing, as well as for quality control in the pharmaceutical industry.

\section{ACKNOWLEDGEMENTS}

The investigation was made using support of the Russian Science Foundation (Grant 14-21-00081).

We give thanks to Prof. Xi-Cheng Zhang (University of Rochester, NY, USA), Prof. Sheng Lan (South China Normal University, Guangzhou, China), Dr. Qingli Zhou and Prof. Cunlin Zhang (Capital Normal University, Beijing, China) for providing us the data used in the paper.

\section{References}

[1] J. F. Federici, B. Schulkin, F. Huang, D. Gary, R. Barat, F. Oliveira, and D. Zimdars, "THz imaging and sensing for security applications - explosives, weapons and drugs," Semicond. Sci. Tech. 20, S266-S280 (2005).

[2] C. Baker, T. Lo, W. R. Tribe, B. E. Cole, M. R. Hogbin, and M. C. Kemp, "Detection of concealed explosives at a distance using terahertz technology," Proc. IEEE 95, 1559-1565 (2007).

[3] M. C. Kemp, "Screening mail for powders using terahertz technology," Proc. SPIE 8189, 81890J (2011).

[4] R. Beigang, S. G. Biedron, S. Dyjak, F. Ellrich, W. Magnus, M. W. Haakestad, D. Hübsch, T. Kartaloglu, et al., "Comparison of terahertz technologies for detection and identification of explosives," Proc. SPIE 9102, 91020C (2014).

[5] G. Katz, S. Zybin, W. A. Goddard, Y. Zeiri, and R. Kosloff, "Direct MD simulations of terahertz absorption and $2 \mathrm{D}$ spectroscopy applied to explosive crystals," J. Phys. Chem. Lett. 5, 772-776 (2014).

[6] M. R. Leahy-Hoppa, M. J. Fitch, X. Zheng, L. M. Hayden, and R. Osiander, "Wideband terahertz spectroscopy of explosives," Chem. Phys. Lett. 434, 227-230 (2007).

[7] A. G. Davies, A. D. Burnett, W. Fan, E. H. Linfield, and J. E. Cunningham, "Terahertz spectroscopy of explosives and drugs," Mater. Today 11, 18-26 (2008).

[8] K. Choi, T. Hong, K. I. Sim, T. Ha, B. C. Park, J. H. Chung, S. G. Cho, et al., "Reflection terahertz time-domain spectroscopy of RDX and HMX explosives," J. Appl. Phys. 115, 023105 (2014).

[9] J. Chen, Y. Chen, H. Zhao, G. J. Bastiaans, and X.-C. Zhang, “Absorption coefficients of selected explosives and related compounds in the range of 0.1-2.8 THz," Opt. Express 15, 12060-12067 (2007).

[10] M. C. Kemp, "Explosives detection by terahertz spectroscopya bridge too far?," IEEE Trans. Terahertz Sci. Technol. 1, 282-292 (2011).

[11] W. Xiong, and J. Shen, "Fingerprint extraction from interference destruction terahertz spectrum," Opt. Express 18, 21798-21803 (2010).

[12] N. Palka, "Identification of concealed materials, including explosives, by terahertz reflection spectroscopy," Opt. Eng. 53, 031202 (2013).

[13] M. Ortolani, J. SD. Lee, U. Schade, and H.-W. Hübers, "Surface roughness effects on the terahertz reflectance of pure explosive materials," Appl. Phys. Lett. 93, 081906 (2008).

[14] W. Withayachumnankul, B. M. Fischer, and D. Abbott, "Numerical removal of water vapor effects from terahertz time-domain spectroscopy measurements," P. Roy. Soc. A-Math. Phy. 464, 2435-2456 (2008). 
[15] A. D. van Rheenen, and M. W. Haakestad, "Detection and identification of explosives hidden under barrier materials - what are the THz-technology challenges?," Proc. SPIE 8017, 801719 (2011)

[16] M. A. Startsev, and A. Y. Elezzabi, "Terahertz frequency continuous-wave spectroscopy and imaging of explosive substances," ISRN Optics 2013, 419507 (2013).

[17] J. B. Sleiman, J. El Haddad, J. B. Perraud, L. Bassel, B. Bousquet, N. Palka, and P. Mounaix, "Qualitative and quantitative analysis of explosives by terahertz time-domain spectroscopy: application to imaging," in Proceedings to the $39^{\text {th }}$ International Conference on IRMMW-THz, 1-2 (IEEE, Tucson, 2014).

[18] U. Puc, A. Abina, M. Rutar, A. Zidanšek, A. Jeglič, and G. Valušis, "Terahertz spectroscopic identification of explosive and drug simulants concealed by various hiding techniques," Appl. Optics 54, 4495-4502 (2015).

[19] K. Kawase, T. Shibuya, S. I. Hayashi, and K. Suizu, "THz imaging techniques for nondestructive inspections," C. R. Phys. 11, 510-518 (2010).

[20] Y. C. Shen, "Terahertz pulsed spectroscopy and imaging for pharmaceutical applications: a review," Int. J. Pharm. 417, 48-60 (2011).

[21] T. Li, A. Patz, L. Mouchliadis, J. Yan, T. A. Lograsso, I. E. Perakis, and J. Wang, "Femtosecond switching of magnetism via strongly correlated spin-charge quantum excitations," Nature 496, 69-73 (2013).

[22] L. Luo, I. Chatzakis, A. Patz, J. Wang, "Ultrafast terahertz probes of interacting dark excitons in chirality-specific semiconducting single-walled carbon nanotubes," Phys. Rev. Lett. 114, 107402 (2015).

[23] V. A. Trofimov and S. A. Varentsova, "New method for analysis of temporal dynamics of medium spectrum under the action of terahertz pulse," Proc. SPIE 6537, 653703 (2007).

[24] V. A. Trofimov, and S. A. Varentsova, "2D THz signature for substance identification," Proc. SPIE 7687, 768707 (2010).

[25] V. A. Trofimov, S. A. Varentsova, J. Shen, C. Zhang, Q. Zhou, and Y. Shi, "2D signature for identification of drugs," Proc. SPIE 8040, 804007 (2011).

[26] V. A. Trofimov, S. A. Varentsova, N. Palka, M. Szustakowski, and T. Trzcinski, "The method of the spectral dynamics analysis of reflected signal for problem of identification of substance," Proc. SPIE 8023, 802300 (2011).

[27] V. A. Trofimov, N. V. Peskov, and D. A. Kirillov, "Efficiency of using correlation function for estimation of probability of substance detection on the base of THz spectral dynamics," Proc. SPIE 8496, 84960T (2012).

[28] V. A. Trofimov, S. A. Varentsova, M. Szustakowski, and N. Palka, "Influence of surface of explosive on its detection and identification using the SDA method for analysis of the reflected THz signal," Proc. SPIE 8734, 87340Q (2013).

[29] V. A. Trofimov, S. A. Varentsova, V. V. Trofimov, and V. V. Tikhomirov, "Peculiarities of the detection and identification of substance at long distance," Proc. SPIE 9102, 910203 (2014).
[30] V. A. Trofimov, S. A. Varentsova, and V. V. Trofimov, "Transmission of THz pulse with a few circles through opaque samples placed at Iong distance (4-6 metres)," Proc. SPIE 9199, 91990E (2014).

[31] V. A. Trofimov, and S. A. Varentsova, "Real-time criteria based on spectral dynamics of medium response for the detection and identification of substance using THz signal," Proc. SPIE 9253, 925305 (2014).

[32] V. A. Trofimov, S. A. Varentsova, and V. V. Trofimov, "Possibility of the detection and identification of substance at long distance at using broad THz pulse," Proc. SPIE 9253, 925308 (2014).

[33] V. A. Trofimov, and S. A. Varentsova, "Efficiency of using the spectral dynamics for pulsed THz spectroscopy of both explosives and other materials," Proc. SPIE 9454, 945409 (2015).

[34] V. A. Trofimov, and S. A. Varentsova, "An effective method for substance detection using the broad spectrum THz signal: a 'terahertz nose'," Sensors 15, 12103-12132 (2015).

[35] H. Y. Liu, Q. F. Dai, L. J. Wu, S. Lan, V. A. Trofimov, and S. A. Varentsova, "Effects of p-type doping on the optical properties of InAs/GaAs quantum dots," Solid State Commun. 152, 435-439 (2012).

[36] J.-H. Sun, J.-L. Shen, L.-S. Liang, X.-Y. Xu, H.-B Liu, and C.-L. Zhang, "Experimental investigation on terahertz spectra of amphetamine type stimulants," Chin. Phys. Lett. 22, 3176-3178 (2005).

[37] A. Rice, Y. Jin, X. F. Ma, X.-C. Zhang, D. Bliss, J. Larkin, and

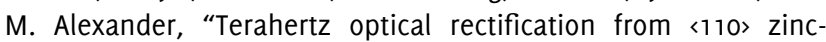
blende crystals," Appl. Phys. Lett. 64, 1324-1326 (1994).

[38] Q. Wu, M. Litz, and X.-C. Zhang, "Broadband detection capability of ZnTe electro-optic field detectors," Appl. Phys. Lett. 68, 2924-2926 (1996).

[39] J. Dai, J. Zhang; W. Zhang, and D. Grischkowsky, "Terahertz timedomain spectroscopy characterization of the far-infrared absorption and index of refraction of high-resistivity, float-zone silicon," J. Opt. Soc. Am B 21, 1379-1386 (2004).

[40] V. A. Trofimov, S. A. Varentsova, V. V. Trofimov, and V. V. Tikhomirov, "High effective algorithm of the detection and identification of substance using the noisy reflected THz pulse," Proc. SPIE 9585, 95850l (2015).

[41] V. A. Trofimov, S. A. Varentsova, I. G. Zakharova, and D. Y. Zagursky, "Principal limitation of standard THz time-domain spectroscopy method of the detection and identification of substance and way of its overcoming," Proc. SPIE 9652, 96520B (2015).

[42] http://www.riken.jp/THzdatabase

[43] http://www.spectra-physics.com/products/ultrafast-lasers/ mai-tai\#specs

[44] http://www.coherent.com/products/?873/Verdi-Family-of-Lasers. 